\title{
RUÍDO NO ALOJAMENTO CONJUNTO: PERCEPÇÃO DAS USUÁRIAS E DOS PROFISSIONAIS DE ENFERMAGEM
}

\author{
Noise in a rooming-in ambience: \\ perception of users and nursing professionals
}

\author{
Liane Fuhr Pivatto(1), Cláudia Giglio de Oliveira Gonçalves(2)
}

\begin{abstract}
RESUMO
Objetivo: mensurar os níveis de ruído e levantar a percepção dos profissionais de enfermagem e usuárias de Alojamento Conjunto em uma maternidade de referência para gestação de alto risco em Curitiba-PR. Métodos: estudo transversal, quantitativo, realizado em alojamento conjunto de um hospital público, no qual a percepção foi levantada por meio de entrevistas com 19 profissionais e 77 usuárias, e o ruído foi avaliado por meio de Medidor Integrador marca Bruel e Kjaer tipo 2230 calibrado na compensação "A". Resultados: os níveis de ruído em todas as áreas do Alojamento Conjunto estão acima do recomendado pela literatura (35 a $45 \mathrm{~dB}(\mathrm{~A})$ para ambiente hospitalar). Os valores mais expressivos foram de $67,7 \mathrm{~dB}(\mathrm{~A})$ no posto de enfermagem e $65,3 \mathrm{~dB}(\mathrm{~A})$ na sala de visitas. Usuárias e profissionais de enfermagem estão expostos a fontes de ruído advindos do processo de trabalho da equipe de saúde, do comportamento dos usuários, dos funcionários e de ruído externo da maternidade. Estratégias para diminuir o ruído e melhorar a ambiência do AC estão voltadas para ações relacionadas ao processo de trabalho da equipe de saúde, a confortabilidade e a gestão. Conclusão: o ruído do Alojamento Conjunto está acima do preconizado. Profissionais de enfermagem percebem mais o ruído externo, enquanto as usuárias mais o ruído interno. Constatou-se que o ruído elevado interfere na ambiência do $\mathrm{AC}$ e afeta as atividades diárias dos profissionais e pacientes.
\end{abstract}

DESCRITORES: Obstetrícia; Efeitos do Ruído; Alojamento Conjunto; Saúde Ocupacional

\section{INTRODUÇÃO}

O ruído está presente nas maternidades, as quais deveriam ser ambientes silenciosos e acolhedores. Estudos sobre ruídos em Unidades Neonatais constatam que as tecnologias incorporadas para cuidar dos recém-nascidos contribuem para a sobrevivência das crianças, mas quando o barulho de instrumentos se une aos choros dos bebês, às vozes dos adultos e à entrada e saída de pessoas, constitui uma situação de poluição sonora $^{1-4}$. Níveis elevados de ruído podem causar perda da audição, aumento da pressão arterial, irritabilidade, tensão, baixa do desempenho e interferência nas comunicações orais ${ }^{5}$.

(1) Universidade Tuiuti do Paraná - UTP, Curitiba, Paraná, Brasil.

(2) Universidade Tuiuti do Paraná - UTP, Curitiba, Paraná, Brasil.

Conflito de interesses: inexistente
Nos Alojamentos Conjuntos (ACs), sistema hospitalar em que o recém-nascido sadio, logo após o nascimento, permanece ao lado da mãe 24 horas por dia, apesar dos bebês não terem como impor modificações na sonoridade ambiental, são dotados de competência comportamental que lhes "permite expressar conforto ou desconforto". Esse comportamento pode sensibilizar os profissionais de saúde a introduzir mudanças no cotidiano do processo de trabalho. ${ }^{2}$

O ruído nas maternidades é apontado como fator de risco para a privacidade e o sucesso na amamentação ${ }^{6}$, para o desenvolvimento do recém-nascido, e também para a saúde das gestantes e recuperação das puérperas ${ }^{4,7}$. A interrupção do sono e do repouso, muitas vezes causada pelo ruído, pode influenciar negativamente no processo de recuperação da saúde da criança ${ }^{8-10}$. A permanência do $\mathrm{RN}$ em um local ruidoso, por período maior que 48 horas, é considerada fator de risco para déficit auditivo ${ }^{11}$. 
Elevados níveis de ruído (acima de $85 \mathrm{~dB}(\mathrm{~A})$ ) são considerados risco ocupacional para os profissionais de saúde. Esses níveis podem causar perda da audição e influenciar na saúde, afetando a vida dos trabalhadores e o processo de trabalho, ${ }^{8,12,13}$. Além disso, o ambiente ruidoso aumenta a carga psíquica negativa do profissional de enfermagem, consequente dos esforços de vigilância, atenção e de sofrimento que podem ser manifestado em queixas de fadiga, insônia e irritabilidade, tensões e dores de cabeça, lapsos de memória e maior esforço mental na realização das tarefas, expondo este profissional a riscos de acidentes e de erros técnicos na execução de suas atividades ${ }^{14}$.

Gestantes expostas a altos níveis de ruído podem apresentar efeitos nocivos à saúde, dentre eles a irritabilidade e a dificuldade de concentração e alteração nos níveis pressórios ${ }^{15}$. Frente ao ruído, existe a possibilidade de aceleração cardíaca, não somente no corpo da gestante, mas também no coração do feto, com diminuição do volume circulatório, vasoconstrição periférica, ampliação da viscosidade do sangue, hipertensão arterial, podendo, inclusive, alterar a posição do feto e complicar o trabalho de parto ${ }^{16,17}$. Complicações na gestação podem ser causadas ou agravadas pelo ruído, como hipertensão, hiperemese gravídica, parto prematuro e nascimento de bebês de baixo peso ${ }^{18}$.

Frente a essas possíveis complicações, um ambiente acolhedor pode ser determinante para o bem-estar dos pacientes e profissionais. Ao falar de acolhimento pressupõe-se a criação de espaços de escuta, de recepção que proporcionem a interação de usuário e trabalhador, que seja acolhedora também no sentido de conforto produzido pela iluminação, cores e sons. É nesse sentido que o Ministério da Saúde lançou a política da ambiência: a qualificação do ambiente hospitalar é determinante no tratamento/recuperação dos pacientes e no desempenho das atividades dos profissionais de saúde ${ }^{19,20}$. A Política Nacional de Humanização da Atenção e da Gestão do SUS - HUMANIZA SUS, em 2003, instituiu a ambiência hospitalar como estratégia para alcançar a qualificação da atenção e da gestão em saúde no SUS. Esta política enfatiza a qualidade do serviço e valoriza não apenas os procedimentos técnicos, mas também os aspectos voltados ao tratamento do ambiente físico, entendido como espaço social, profissional e de relações interpessoais, que deve proporcionar atenção acolhedora e resolutiva como também humana $^{19,20}$. O ruído esta inserido dentro dessa política.

Pesquisas sobre ruído em Alojamento Conjunto ainda são incipientes. Foram encontrados poucos estudos que expressem as condições acústicas ou que tratem da análise do ruído em maternidade referência para gestação de risco. Assim, o objetivo desta pesquisa é mensurar e analisar o ruído na ambiência de um Alojamento Conjunto e levantar a percepção dos efeitos sobre a saúde relatada por usuárias e profissionais de enfermagem.

\section{MÉTODOS}

O projeto foi submetido ao Comitê de Ética de Pesquisas em Seres Humanos do HC/ UFPR sob número 2256.150/2010-06 e aprovado (CAAE:0150.0.208.000-10). Foi utilizado o Termo de Consentimento Informado Livre e Esclarecido, no qual constam todas as orientações necessárias para a participação na pesquisa, antes de quaisquer procedimentos.

Trata-se de um estudo transversal, com abordagem quantitativa, desenvolvido num hospital público, no Alojamento Conjunto, que é referência do SUS para gestação de alto risco do Município de Curitiba/PR. Possui 28 leitos para atender gestantes, puérperas e recém-nascidos, sendo 16 leitos da unidade $A C$, seis leitos de gestantes em tratamento clínico e seis leitos para puérperas cujos recém-nascidos estavam internados na Unidade de Terapia Intensiva Neonatal (UTIN).

Os sujeitos da pesquisa foram 77 usuárias (puérperas e gestantes), 38 mães de recém-nascidos e 19 profissionais de enfermagem.

A amostra das usuárias (gestantes e puérperas) foi intencional, limitada pelo período do estudo estipulado de fevereiro a março de 2011. Do total de 328 usuárias atendidas neste período, 81 participaram do estudo, o que corresponde a $23,47 \%$ do total, sendo que quatro usuárias foram excluídas por intercorrências clínicas que impossibilitaram a conclusão da entrevista. Destas, 38 mães foram selecionadas para também responder a um questionário com questões relativas ao comportamento do recém-nascido frente ao ruído do Alojamento Conjunto. Os 19 profissionais de enfermagem que participaram do estudo correspondem a 100\% dos servidores lotados no Alojamento Conjunto no período do estudo.

Os critérios de inclusão dos profissionais de enfermagem foram: vínculo empregatício com a instituição e aceitar participar da pesquisa. Para a inclusão das usuárias: ser gestante e/ou puérpera concordante com a pesquisa. No caso dos recém-nascidos, a mãe deveria aceitar responder o questionário aplicado em forma de entrevista pela pesquisadora.

Os dados sobre percepção do ruído e seus efeitos na saúde foram pesquisados no período de fevereiro a março de 2010, utilizando três 
questionários distintos elaborados pela autora com questões abertas e semi-abertas, sendo: um para coleta de dados de recém-nascidos, um para as gestantes e puérperas e outro para profissionais de enfermagem.

No questionário aplicado para as mães dos $\mathrm{RN}$ constaram perguntas relativas às condições do nascimento (peso, idade gestacional ao nascer, tipo de parto) e questões relativas ao comportamento do recém-nascido frente ao ruído ambiental do Alojamento Conjunto. Tomou-se cuidado para realizar as entrevistas com as mães quando não estavam em amamentação, prestando cuidados gerais ao bebê e nos horários de alimentação e repouso.

No questionário aplicado às gestantes e puérperas, constaram questões relativas à percepção do ruído no ambiente do Alojamento Conjunto e seus efeitos na saúde, horários de ruído mais intenso, principais fontes de ruído, medidas para diminuir o ruído na unidade e perguntas sobre queixas de saúde, riscos gestacionais, ocupação, profissão e procedência. No questionário dos profissionais de enfermagem, foram abordados dados sociodemográficos, formação e prática profissional,queixas de saúde e questões relativas à percepção do ruído e efeitos na saúde.

A mensuração do ruído foi realizada no período de fevereiro a setembro de 2011, com apoio de um profissional da Engenharia, por meio de um medidor de nível de pressão sonora, conforme as recomendações da NBR-10152/1987, a qual define os níveis de pressão sonora para conforto acústico em diferentes áreas do hospital. Também foram considerados os critérios legais estabelecidos pela legislação brasileira, Portaria n. ${ }^{\circ} 3214 / 78$ do Ministério do Trabalho e Emprego, Norma Regulamentadora NR-17, Ergonomia e a NBR-10152/200021. O equipamento utilizado foi o Medidor Integrador Portado pelo avaliador, marca Bruel \& Kjaer, tipo 2230, devidamente calibrado. Para avaliação dos níveis de pressão sonora, operou-se na curva de compensação "A", sendo realizadas várias medições, nos períodos da manhã, tarde e noite, medindo-se os níveis mínimos, máximos, e o nível médio fornecido pelo recurso de medição de nível equivalente (Leq) em salas da unidade de Alojamento Conjunto.

Os resultados da percepção do ruído dos profissionais de enfermagem e usuárias foram tabulados e apresentados em tabelas. As questões abertas referentes às ações que podem contribuir com a ambiência do Alojamento Conjunto, segundo a opinião dos profissionais de enfermagem e usuárias, foram agrupadas nos três eixos articuladores da ambiência, conforme a definição do Ministério da Saúde: medidas relacionadas ao processo de trabalho, aos elementos do ambiente que interagem com o homem, e medidas relacionadas com a otimização de recursos para o atendimento humanizado e acolhedor nos serviços de saúde.

Os resultados da mensuração do ruído (em $\mathrm{dB}(\mathrm{A})$ ) foram tabulados de acordo com as frequências obtidas e apresentados em quadro, para comparação e análise.

Para as análises estatísticas foram utilizados métodos de Estatística Descritiva (tabelas de frequências) e de Inferência Estatística (teste de diferença de proporções). Realizou-se a análise comparativa da percepção do ruído pelas puérperas e profissionais com os dados da mensuração do ruído. Os neonatos não foram submetidos à triagem neonatal.

\section{RESULTADOS}

Os resultados das medições dos níveis de ruído em $\mathrm{dB}(\mathrm{A})$ das diversas áreas do Alojamento Conjunto (Figura 1) evidenciam níveis de pressão sonora acima do recomendado pela Legislação Brasileira (35 a $45 \mathrm{~dB}(\mathrm{~A})$ para o ambiente hospitalar. Os valores mais expressivos foram de $67,5 \mathrm{~dB}(\mathrm{~A})$ no posto de enfermagem, no período da manhã; e na sala de visitas, no período da tarde, de 65,3 $d B(A)$. 


\begin{tabular}{|c|c|c|c|c|c|}
\hline SETOR & PERÍODO & $\begin{array}{c}\text { RUÍDO } \\
\text { conTíNUO } \\
\text { Leq dB(A) }\end{array}$ & MÍN & MÁX & $\begin{array}{l}\text { DURAÇÃO } \\
\text { AVALIAÇÃOO }\end{array}$ \\
\hline \multirow{3}{*}{ Secretaria } & Manhã & 64,5 & 48,0 & 86,8 (carrinho) & 1 hora \\
\hline & Tarde & 63,7 & 50,6 & $\begin{array}{c}96,2 \text { (batida de } \\
\text { porta) }\end{array}$ & $30 \mathrm{~min}$ \\
\hline & Noite & 58,7 & 44,8 & 71,3 & $20 \mathrm{~min}$ \\
\hline \multirow{2}{*}{$\begin{array}{c}\text { Posto de } \\
\text { Enfermagem }\end{array}$} & Manhã & 67,5 & 47,9 & $\begin{array}{c}84,9 \text { (batida de } \\
\text { porta) }\end{array}$ & $30 \mathrm{~min}$ \\
\hline & Noite & 58,0 & 40,9 & 60,0 & $20 \mathrm{~min}$ \\
\hline \multirow{3}{*}{ Corredor } & Manhã & 62,2 & 49,5 & 83 & $30 \mathrm{~min}$ \\
\hline & Tarde & 63,2 & 46,9 & $\begin{array}{c}85,3 \text { (porta } \\
\text { batendo) }\end{array}$ & $30 \mathrm{~min}$ \\
\hline & Noite & 62,3 & 47,6 & 77,8 & $20 \mathrm{~min}$ \\
\hline \multirow{3}{*}{$\begin{array}{c}\text { Enfermaria } 218 \\
\text { (5 leitos, mãe e } \\
\text { bebê) }\end{array}$} & Manhã & 61,6 & 44,0 & $\begin{array}{l}82,0 \text { (batidas de } \\
\text { porta do quarto } \\
\text { e bwc) }\end{array}$ & 1 hora \\
\hline & Tarde & 53,0 & 46,6 & 69,4 & $30 \mathrm{~min}$ \\
\hline & Noite & 61,9 & 37,5 & 81,5 & $20 \mathrm{~min}$ \\
\hline \multirow{3}{*}{$\begin{array}{c}\text { Enfermaria } \\
206 \text { - Sala da } \\
\text { Pediatria }\end{array}$} & Manhã & 48,6 & 41,3 & 64,5 & $20 \mathrm{~min}$ \\
\hline & Tarde & 56,4 & 50,6 & 68,6 & $20 \mathrm{~min}$ \\
\hline & Noite & 45,6 & 40,9 & 60,0 & $20 \mathrm{~min}$ \\
\hline $\begin{array}{c}\text { Hall } \\
\text { (com visitas) } \\
16 \text { às } 17 \mathrm{~h}\end{array}$ & Tarde & 65,3 & 52,4 & 83,0 & $30 \mathrm{~min}$ \\
\hline \multirow{2}{*}{$\begin{array}{c}\text { Hall } \\
\text { (sem visitas) }\end{array}$} & Tarde & 61,5 & 50,6 & 75,6 & $30 \mathrm{~min}$ \\
\hline & Noite & 57,2 & 49,8 & 76,6 & $20 \mathrm{~min}$ \\
\hline
\end{tabular}

Figura 1 - Níveis de ruído nas áreas do alojamento conjunto

Em relação ao perfil das usuárias, verificou-se que $83,12 \%$ são puérperas casadas $(42,86 \%)$, estão na idade reprodutiva $(77,92 \%)$ e possuem ensino fundamental completo $(42,86 \%)$. Os riscos gestacionais mais encontrados foram hipertensão $(27,27 \%)$, diabetes gestacional e aborto $(20,77 \%$ cada) e doenças infecciosas com (12,98\%). Quanto aos profissionais da enfermagem, há $89,47 \%$ profissionais do sexo feminino, o predomínio de idade é entre 50 e 59 anos (36,84\%), 42,11\% tem formação de nível superior e $63,16 \%$ trabalham a menos de cinco anos no serviço.

A Tabela 1 traz os riscos gestacionais apresentados pelas usuárias, segundo classificação do Ministério da Saúde: características individuais e condições sociodemográficas desfavoráveis, a história reprodutiva anterior, as doenças obstétricas na gravidez atual e as intercorrências clínicas. O grupo das doenças obstétricas na gravidez atual apresentou maior número de casos (total de 73).

Quanto aos recém-nascidos, a grande maioria nasceu com peso superior a $2500 \mathrm{~g}$ e de gestação a termo. Segundo o relato das mães sobre os comportamentos dos RNs em relação ao ruído, $73,68 \%$ mencionam que os RNs dormem mais no período diurno, $39,47 \%$ observaram choro mais intenso à noite e $68,42 \%$ das mães relataram que os RNs se assustam com os ruídos do ambiente do Alojamento Conjunto. 
Tabela 1 - Caracterização das usuárias do alojamento conjunto em relação aos riscos gestacionais agrupados segundo o ministério da saúde $(\mathrm{N}=77)$

\begin{tabular}{|c|c|c|}
\hline $\begin{array}{l}\text { GRUPO DE FATOR DE RISCO } \\
\text { GESTACIONAL }\end{array}$ & FREQUÊNCIA ABSOLUTA & FREQUÊNCIA RELATIVA \\
\hline \multicolumn{3}{|l|}{ 1- CARACTERÍSTICAS INDIVIDUAIS } \\
\hline Drogadição & 5 & 6,49 \\
\hline Tabagismo & 5 & 6,49 \\
\hline Idade & 2 & 2,6 \\
\hline Etilismo & 2 & 2,6 \\
\hline Depressão & 2 & 2,6 \\
\hline Obesidade & 1 & 1,3 \\
\hline \multicolumn{3}{|c|}{ 2- HISTÓRIA REPRODUTIVA ANTERIOR } \\
\hline Aborto & 16 & 20,77 \\
\hline CST Eletiva & 1 & 1,3 \\
\hline Antecedentes Obstétricos & 1 & 1,3 \\
\hline Multiparidade & 1 & 1,3 \\
\hline \multicolumn{3}{|l|}{ 3- INTERCORRÊNCIAS CLÍNICAS } \\
\hline Doenças Infecciosas & 10 & 12,98 \\
\hline Insuficiência Renal & 6 & 7,79 \\
\hline Convulsão & 4 & 5,19 \\
\hline Cardiopatia & 4 & 5,19 \\
\hline Alterações na Tireóide & 2 & 2.6 \\
\hline AVC & 2 & 2,6 \\
\hline Linfoma & 1 & 1,3 \\
\hline \multicolumn{3}{|c|}{ 4- DOENÇAS OBSTÉTRICAS NA GRAVIDEZ ATUAL } \\
\hline Hipertensão & 21 & 27,27 \\
\hline Diabetes gestacional & 16 & 20.77 \\
\hline Hemorragias & 10 & 12,98 \\
\hline $\begin{array}{l}\text { Alterações no desenvolvimento do } \\
\text { feto }\end{array}$ & 7 & 9,09 \\
\hline Alterações no líquido amniótico & 5 & 6,49 \\
\hline Gestação múltipla & 5 & 6,49 \\
\hline Trabalho de parto prematuro & 2 & 2,59 \\
\hline Anemia severa & 2 & 2,59 \\
\hline Distúrbios da coagulação & 2 & 2,59 \\
\hline Cisto uterino & 1 & 1,29 \\
\hline Gigantomastia & 1 & 1,29 \\
\hline Hiperêmese & 1 & 1,29 \\
\hline
\end{tabular}

Na Tabela 2 são apresentados os dados relativos à percepção do ruído externo e interno pelos profissionais de enfermagem e usuárias do ambiente de Alojamento Conjunto.

O nível de ruído proveniente do ambiente externo não foi mensurado nesse estudo. Em relação à percepção, foi considerado alto por $84,21 \%$ dos profissionais de enfermagem e por $49,35 \%$ das usuárias. Já o ruído interno máximo mensurado $(96,2 \mathrm{~dB}(\mathrm{~A})$ oriundo do barulho provocado pela batida das portas) é considerado alto por $62,34 \%$ das usuárias e $47,37 \%$ dos profissionais de enfermagem. 
Tabela 2 - Percepção dos profissionais de enfermagem e das usuárias sobre o ruído externo e interno do alojamento conjunto $(\mathrm{N}=96)$

\begin{tabular}{lcccc}
\hline \multirow{2}{*}{ PERCEPÇÃo } & \multicolumn{2}{c}{$\begin{array}{c}\text { PROFISSIONAIS DE SAÚDE } \\
(\mathbf{n}=\mathbf{1 9})\end{array}$} & \multicolumn{2}{c}{ USUÁRIAS $(\mathbf{n}=\mathbf{7 7})$} \\
\cline { 2 - 5 } & FREQ ABS. & FREQ. REL. & FREQ ABS. & FREQ. REL. \\
\hline Ruído EXTERNO & 16 & 84,21 & 38 & 49,35 \\
Alto & 2 & 5,26 & 22 & 28,57 \\
Médio & 1 & 10,53 & 16 & 20,78 \\
Baixo & 0 & 0 & 1 & 1,30 \\
Sem resposta & & & & \\
\hline Ruído INTERNO & 9 & 47,37 & 48 & 62,34 \\
Alto & 9 & 47,37 & 16 & 20,78 \\
Médio & 1 & 5,26 & 13 & 16,88 \\
Baixo & & &
\end{tabular}

A Tabela 3 apresenta as principais fontes de ruído do Alojamento Conjunto, segundo a percepção dos entrevistados.

As principais fontes de ruído (78,95\%), na visão dos profissionais de enfermagem, são a conversação e o telefone. Tal percepção vem ao encontro dos níveis de ruído mensurados nesse estudo: $67,5 \mathrm{~dB}(\mathrm{~A})$ no posto de enfermagem. Já na opinião das usuárias as fontes mais significantes de ruído são as batidas das portas $(68,94 \%)$ e o choro dos bebês (64,94\%). Essa percepção também é coerente quando comparada ao nível máximo de ruído encontrado nas enfermarias: $82,0 \mathrm{~dB}(\mathrm{~A})$.

A Tabela 4 apresenta o período em que o ruído é considerado mais intenso no Alojamento Conjunto.

Tabela 3 - Percepção dos profissionais de enfermagem e das usuárias das fontes de ruído no alojamento conjunto $(\mathrm{N}=96)$

\begin{tabular}{|c|c|c|c|c|}
\hline \multirow{2}{*}{$\begin{array}{l}\text { PERCEPÇÃO DAS } \\
\text { FONTES DE RUÍDO }\end{array}$} & \multicolumn{2}{|c|}{$\begin{array}{l}\text { PROFISSIONAIS DE SAÚDE } \\
\qquad(n=19)\end{array}$} & \multicolumn{2}{|c|}{ USUÁRIAS ( $\mathrm{n}=77$ ) } \\
\hline & FREQ ABS. & FREQ. REL. & FREQ ABS. & FREQ. REL. \\
\hline Conversação & 15 & 78,95 & 47 & 61,04 \\
\hline Bebês & 11 & 57,89 & 50 & 64,94 \\
\hline Barulho das portas & 10 & 52,63 & 53 & 68,83 \\
\hline $\begin{array}{l}\text { Atividades dos } \\
\text { profissionais em geral }\end{array}$ & 12 & 63,16 & 41 & 53,25 \\
\hline Telefone & 15 & 78,95 & 20 & 25,97 \\
\hline Visita dos familiares & 9 & 47,37 & 21 & 27,27 \\
\hline Visita médica & 8 & 42,11 & 19 & 24,68 \\
\hline $\begin{array}{l}\text { Passagem de plantão da } \\
\text { enfermagem }\end{array}$ & 4 & 21,05 & 3 & 3,90 \\
\hline $\begin{array}{l}\text { Barulho dos instrumentos } \\
\text { em uso }\end{array}$ & 5 & 26,32 & 40 & 51,95 \\
\hline Rádio & 6 & 31,58 & 3 & 3,90 \\
\hline Campainha & 9 & 47,37 & - & - \\
\hline $\begin{array}{l}\text { Líderes de religião oram } \\
\text { muito alto }\end{array}$ & 1 & 5,26 & - & - \\
\hline
\end{tabular}


Tabela 4 - Período em que o ruído é mais intenso no alojamento conjunto segundo os profissionais de enfermagem e usuárias

\begin{tabular}{|c|c|c|c|c|}
\hline \multirow[t]{2}{*}{ PERÍODO DO DIA } & \multicolumn{2}{|c|}{$\begin{array}{l}\text { PROFISSIONAIS DE SAÚDE } \\
(n=19)\end{array}$} & \multicolumn{2}{|c|}{ USUÁRIAS (n = 77) } \\
\hline & FREQ ABS. & FREQ. REL. & FREQ ABS. & FREQ. REL. \\
\hline Manhã & 9 & 47,37 & 40 & 51,95 \\
\hline Tarde & 4 & 21,05 & 11 & 14,29 \\
\hline Noite & 3 & 15,79 & 10 & 12,99 \\
\hline Dia inteiro & 1 & 5,26 & 15 & 19,48 \\
\hline Sem resposta & 2 & 10,53 & - & - \\
\hline
\end{tabular}

Na opinião de 51,95\% das usuárias e de 47,37\% dos profissionais de enfermagem, é no período da manhã que o ambiente do Alojamento Conjunto tem ruído mais elevado

Na percepção do ambiente em relação ao ruído, mais da metade dos profissionais $(52,64 \%)$ alega que o exercício de suas atividades profissionais não gera ruído. Já $50,65 \%$ das usuárias consideram que seu comportamento gera ruído e que este afeta a saúde dos recém-nascidos. Usuárias e profissionais de enfermagem são unânimes em afirmar que o ruído elevado prejudica a saúde dos pacientes. Não foram observadas diferenças estatisticamente significantes entre as usuárias e os profissionais de enfermagem quanto à percepção do seu comportamento gerar ruído.

A Tabela 5 apresenta as queixas de saúde relatadas pelos sujeitos do estudo.

Tabela 5 - Queixas dos profissionais de enfermagem e usuárias do alojamento conjunto ( $\mathrm{N}=96$ )

\begin{tabular}{|c|c|c|c|c|}
\hline \multirow{2}{*}{ QUEIXAS } & \multicolumn{2}{|c|}{$\begin{array}{l}\text { PROFISSIONAIS DE SAÚDE } \\
\qquad(n=19)\end{array}$} & \multicolumn{2}{|c|}{ USUÁRIAS (n = 77) } \\
\hline & FREQ. ABS. & FREQ. REL. & FREQ. ABS. & FREQ. REL. \\
\hline Cansaço & 12 & 63,16 & 46 & 59,74 \\
\hline Estresse & 9 & 47,37 & - & - \\
\hline Ansiedade & 9 & 47,37 & 65 & 84,42 \\
\hline $\begin{array}{l}\text { Alterações da pressão } \\
\text { sanguínea }\end{array}$ & 9 & 47,37 & 24 & 31,17 \\
\hline Alterações da visão & 7 & 36,84 & 19 & 24,68 \\
\hline Perturbações do sono & 6 & 31,58 & 30 & 38,96 \\
\hline Irritabilidade & 6 & 31,58 & 48 & 62,34 \\
\hline Nervosismo & 5 & 26,32 & 45 & 58,44 \\
\hline Problemas circulatórios & 5 & 26,32 & - & - \\
\hline Problemas cardíacos & 4 & 21,05 & - & - \\
\hline $\begin{array}{l}\text { Problemas } \\
\text { gastrointestinais }\end{array}$ & 4 & 21,05 & 32 & 41,56 \\
\hline Incômodo & 4 & 21,05 & - & - \\
\hline Zumbidos & 3 & 15,79 & 23 & 29,87 \\
\hline Perda auditiva & 3 & 15,79 & 10 & 12,99 \\
\hline Dor de cabeça constante & 2 & 12,53 & 24 & 31,17 \\
\hline $\begin{array}{l}\text { Dificuldade de } \\
\text { compreender fala }\end{array}$ & 1 & 5,26 & 16 & 20,78 \\
\hline
\end{tabular}

NOTA: Os sujeitos poderiam indicar mais de um problema de saúde. 
A ansiedade e a irritabilidade foram os fatores mais citados pelas usuárias com $84,42 \%$ e $62,34 \%$, respectivamente, enquanto que o cansaço e estresse são as queixas mais relevantes dos profissionais de enfermagem com $63,16 \%$ e $47,37 \%$.
A Tabela 6 mostra as alternativas que podem contribuir na redução do ruído. Profissionais e usuárias acreditam que exercícios de percepção do silêncio e a musicoterapia podem ajudar a minimizar o ruído no Alojamento Conjunto.

Tabela 6 - Estratégias que minimizam o ruído no alojamento conjunto ( $\mathrm{N}=96)$

\begin{tabular}{|c|c|c|c|c|}
\hline \multirow[t]{2}{*}{ SUGESTÕES } & \multicolumn{2}{|c|}{$\begin{array}{l}\text { PROFISSIONAIS DE SAÚDE } \\
(\mathrm{N}=19)\end{array}$} & \multicolumn{2}{|c|}{ USUÁRIAS (N = 77) } \\
\hline & FREQ. ABS. & FREQ. REL. & IEQ. ABS. & FREQ. REL. \\
\hline \multicolumn{5}{|c|}{ EXERCÍCIOS DE PERCEPÇÃO DO SILÊNCIO } \\
\hline Sim & 17 & 89,47 & 74 & 96,10 \\
\hline Não & 1 & 5,26 & - & - \\
\hline Talvez & 1 & 5,26 & - & - \\
\hline Não sei & - & - & 2 & 2,60 \\
\hline Sem resposta & - & - & 1 & 1,30 \\
\hline \multicolumn{5}{|c|}{ MUSICOTERAPIA } \\
\hline Sim & 18 & 94,74 & 60 & 77,92 \\
\hline Não & 1 & 5,26 & 12 & 15,58 \\
\hline Não sei & - & - & 5 & 6,49 \\
\hline
\end{tabular}

A Tabela 7 apresenta as ações que podem contribuir para a ambiência do Alojamento Conjunto, segundo a opinião dos profissionais de enfermagem e usuárias. As ações foram agrupadas levando em consideração os três eixos do conceito de ambiência hospitalar da Política de Humanização da Atenção e da Gestão do SUS do Ministério da Saúde (2003 e 2007).

Ao se comparar as respostas dos profissionais de saúde e das usuárias, por meio do teste de diferença de proporções, verifica-se diferença significante em todas as ações. Nas sugestões sobre Ações Relacionadas ao Processo de Trabalho e Ações Relacionadas à Confortabilidade, a proporção de respostas é significantemente maior entre os profissionais da saúde, enquanto que para as Ações Relacionadas à Gestão, a proporção é significantemente maior entre as usuárias.

\section{DISCUSSÃO}

Os níveis de ruído encontrados neste estudo estão acima dos recomendados (Figura 1), tanto pela NBR-12.179/1992, como pela NBR-10152/2000. A literatura mostra a identificação de níveis também elevados em outros hospitais, ou seja, entre 60 e $70 \mathrm{~dB}(\mathrm{~A})$ na $\mathrm{UCIP}^{13}$; $62,3 \mathrm{~dB}(\mathrm{~A})$ de 55,3 a 72,2 $\mathrm{dB}(\mathrm{A})$ de pressão sonora durante a passagem de plantão e acima de $50 \mathrm{~dB}(\mathrm{~A})$ na $\mathrm{UCIN}^{3} ; 59,5 \mathrm{~dB}(\mathrm{~A})$ numa unidade de cuidado intermediário neonatal ${ }^{4}$. Portanto, já há consenso científico de que os níveis de ruídos em hospitais são elevados, no entando são insuficientes para causar perda auditiva.

Em relação às usuárias do $A C$, a maior parte delas tem idade entre 18 e 35 anos, uma faixa etária em que o risco gestacional costuma ser menos elevado ${ }^{22}$, no entanto apresentaram doenças obstétricas graves. A maioria é casada ou tem união estável. Também houve a prevalência destes fatores em outra pesquisa realizada, existindo algumas semelhanças quanto à idade $\mathrm{e}$ estabilidade familiar desta população ${ }^{23}$.

Os riscos gestacionais das usuárias e que justificam a permanência das mesmas no AC de maternidade referência para gestação de risco foram hipertensão, diabetes, aborto e doenças infecciosas, os quais requerem cuidados maiores. Tal qual foi verificado em hospital público, cuja maternidade também é referência para gestação de risco ${ }^{24}$. Isto demonstra que os fatores que caracterizam esta gestação são os mesmos, porém a frequência com que aparecem pode variar ${ }^{25}$.

Foi constatado - na cidade de São Paulo - que a mortalidade consequente da hipertensão arterial é responsável por $66,9 \%$ de óbitos no puerpério e $34 \%$ no decorrer da gestação, sendo as causas do óbito de $44,4 \%$ o acidente vascular cerebral, de $24,6 \%$ o edema agudo de pulmão, e de $14,1 \%$ as 
Tabela 7 - Ações que podem contribuir com a ambiência do alojamento conjunto segundo os profissionais de enfermagem e as usuárias

\begin{tabular}{|c|c|c|c|c|c|}
\hline \multirow{2}{*}{ RESULTADOS } & \multicolumn{2}{|c|}{$\begin{array}{l}\text { PROFISSIONAIS DE } \\
\text { SAÚDE }(n=19)\end{array}$} & \multicolumn{2}{|c|}{ USUÁRIAS ( $n=77$ ) } & \multirow{2}{*}{$\mathbf{P}$} \\
\hline & $\begin{array}{l}\text { Freq. } \\
\text { Rel. }\end{array}$ & $\begin{array}{l}\text { Freq. } \\
\text { Abs. }\end{array}$ & $\begin{array}{l}\text { Freq. } \\
\text { Rel. }\end{array}$ & $\begin{array}{l}\text { Freq. } \\
\text { Abs. }\end{array}$ & \\
\hline \multicolumn{6}{|l|}{ Ações relacionadas ao processo de trabalho } \\
\hline Exercícios de percepção do silêncio & 19 & 100,00 & 63 & 81,82 & \\
\hline Exercícios de percepção corporal & 16 & 84,21 & - & - & \\
\hline Maior habilidade dos profissionais de saúde & 6 & 31,57 & 62 & 80,52 & \\
\hline Educação dos trabalhadores & 13 & 68,42 & 34 & 31,17 & \\
\hline Educação dos pacientes & 13 & 68,42 & 20 & 25,97 & \\
\hline $\begin{array}{l}\text { Diminuir volume do som dos equipamentos } \\
\text { eletrônicos }\end{array}$ & 5 & 19,31 & 2 & 2,59 & \\
\hline Falar mais baixo & 9 & 47,37 & - & - & \\
\hline Mudar hora do banho do RN & - & - & 2 & 2,59 & \\
\hline Sub Total & 81 & 53,3 & 183 & 29,7 & $0,0000^{*}$ \\
\hline \multicolumn{6}{|l|}{ Ações relacionadas à confortabilidade } \\
\hline $\begin{array}{l}\text { Cuidado ao abrir e fechar as portas das } \\
\text { enfermarias }\end{array}$ & 17 & 89,47 & 48 & 62,34 & \\
\hline Utilizar alarmes luminosos & 15 & 78,95 & - & - & \\
\hline Incentivar a musicoterapia no AC & 17 & 89,47 & - & - & \\
\hline $\begin{array}{l}\text { Incentivar reflexão com prática de atividades } \\
\text { religiosas na rotina de cuidados do serviço. }\end{array}$ & 14 & 73,68 & - & - & \\
\hline Diminuir o trânsito nos entornos do hospital & - & - & 1 & 1,29 & \\
\hline Subtotal & 63 & 66,3 & 49 & 12,7 & $0,0000^{*}$ \\
\hline \multicolumn{6}{|l|}{ Ações relacionadas à gestão } \\
\hline $\begin{array}{l}\text { Realizar manutenção dos materiais e } \\
\text { equipamentos }\end{array}$ & 17 & 89,47 & 74 & 96,10 & \\
\hline Medir ruído periodicamente & 16 & 84,21 & 42 & 54,55 & \\
\hline $\begin{array}{l}\text { Realizar manutenção das portas das } \\
\text { enfermarias do } A C\end{array}$ & 1 & 5,26 & 72 & 93,51 & \\
\hline $\begin{array}{l}\text { Reduzir o número de pacientes por } \\
\text { enfermaria }\end{array}$ & - & - & 43 & 55,84 & \\
\hline $\begin{array}{l}\text { Reduzir o número de pessoas que circulam } \\
\text { no AC diariamente }\end{array}$ & - & - & 43 & 55,84 & \\
\hline Realizar isolamento acústico & 1 & 5,26 & 21 & 27,27 & \\
\hline $\begin{array}{l}\text { Melhorar infra-estrutura (válvulas do } \\
\text { sanitário fazem muito barulho) }\end{array}$ & 2 & 10,52 & 23 & 29,87 & \\
\hline Determinar a lei do silêncio & 2 & 10,52 & 4 & 5,19 & \\
\hline $\begin{array}{l}\text { Ajustes e manutenção do serviço de } \\
\text { hotelaria (carrinhos de alimentos são } \\
\text { barulhentos) }\end{array}$ & - & - & 3 & 3,90 & \\
\hline Colocar placas indicativas de silêncio & 2 & 10,52 & 13 & 16,88 & \\
\hline $\begin{array}{l}\text { Retirar o depósito de gás em frente da } \\
\text { maternidade }\end{array}$ & 2 & 10,53 & 17 & 22,07 & \\
\hline Subtotal & 43 & 20,6 & 355 & 41,9 & $0,0000^{*}$ \\
\hline
\end{tabular}


coagulopatias $^{26}$. Pode-se concluir que as doenças que levam a óbito as mulheres no puerpério são de impacto social ${ }^{27}$, que podem ser prevenidas ou controladas com medidas de atenção ao pré-natal, parto e puerpério.

Em relação à percepção das mães sobre os efeitos do ruído no $\mathrm{RN}$, observou-se que os recém-nascidos dormem mais de dia, e se assustam e se agitam com o ruído do ambiente hospitalar. Constatou-se que frente ao ruído relatam-se reações de isolamento da interação social, alteração do estado de sono e descanso, além de hipóxia, elevação da pressão intracraniana e da pressão sanguínea, apnéia e bradicardia ${ }^{10,28} \mathrm{e}$, nesta pesquisa, talvez tenha alguma relação com a intensidade do choro dos bebês, mais intenso à noite, conforme $39,47 \%$ das usuárias ${ }^{29-31}$.

As usuárias foram unânimes em afirmar que o ruído no Alojamento Conjunto afeta a saúde do bebê, retarda a recuperação dos doentes e prejudica o repouso dos recém-nascidos e das mães. Pesquisa ${ }^{32}$ que estudou as reações do RN frente ao ruído verificou o despertar do sono e o choro (muitas vezes acompanhado de agitação) como forma de expressar desconforto, inquietação e estresse.

O ruído proveniente do ambiente externo é considerado alto pelos profissionais de enfermagem, enquanto que o ruído interno é considerado alto pelas usuárias. Uma hipótese para esse resultado é que ao se defrontar com o ambiente da hospitalização e por estarem fora de seu ambiente natural, as usuárias têm uma percepção negativa do ruído característico do ambiente hospitalar, proveniente em sua grande maioria do processo de trabalho da equipe de saúde. Os profissionais já criaram mecanismos de adaptação e de naturalização dos ruídos da ambiência hospitalar ${ }^{33}$. Além do que, o ruído informativo - a exemplo dos alarmes sonoros e choro de bebês - alerta os profissionais sobre alterações no quadro clínico, podendo ser considerado aliado ao processo de trabalho e influenciar as práticas e a tomada de decisão.

A percepção dos estressores internos depende das características de cada pessoa, envolvendo personalidade, crenças, ansiedades e expectativas. Os estressores externos são as situações externas que interferem na saúde, como iluminação do ambiente de trabalho e o ruído, ou seja, ocorre constantemente uma interação entre o indivíduo e a ambiência, a partir dos contextos específicos ${ }^{34}$.

As principais fontes geradoras do ruído, segundo os profissionais, foram a conversação, as visitas dos familiares e as atividades dos profissionais. Já as usuárias atribuíram o ruído ao barulho das portas e ao choro dos bebês. É possível encontrar uma relação assimétrica da maior fonte de ruído: para os profissionais, as fontes estão ligadas ao processo de trabalho e rotinas de serviço, o que pode ser constatado analisando os níveis de ruído encontrados no posto de enfermagem $(67,5 \mathrm{~dB}(\mathrm{~A}))$ e na sala de visitas $(65,3 \mathrm{~dB}(\mathrm{~A}))$. Para as usuárias, as fontes estão ligadas à postura dos profissionais e à convivência hospitalar na maternidade, percepção condizente ao nível máximo de ruído mensurado na enfermaria $(82.0 \mathrm{~dB}(\mathrm{~A}))$.

Profissionais e usuárias percebem que o ruído é mais intenso no período diurno, confirmando os resultados das medições dos níveis de ruído (Figura 1), como também foi verificado em outros estudos $^{2,13}$. Estudo ${ }^{4}$ constatou como sendo o período noturno o mais ruidoso. Nesta pesquisa, o resultado pode ser atribuído ao ritmo intenso das atividades técnicas da equipe de saúde no período da manhã, como também à grande circulação de pessoas em função das visitas médicas e das práticas clínicas dos alunos que acontecem como rotina pela manhã.

Os efeitos fisiológicos do ruído podem alterar a frequência cardíaca e a pressão sanguínea, bem como influenciar no processo digestivo e de repouso $0^{4,35}$. Trata-se, portanto, de um alerta que o ruído em ambiente hospitalar traz implicações nocivas para a saúde dos usuários e dos trabalhadores.

Exercícios de percepção do silêncio são sugeridos como estratégias que minimizam o ruído no $A C$ pelos profissionais e usuárias. Esta demanda se mostra urgente, conforme o primeiro eixo da ambiência apresentado pelo Ministério da Saúde ${ }^{20}$. O máximo respeito pelo silêncio poderá ser estimulado, aproveitando a oportunidade para cada um se ouvir. A percepção corporal que não é referida por nenhuma usuária, recebe a aprovação dos profissionais de enfermagem. A percepção corporal possibilita ouvir os sons do corpo, a exemplo da respiração, das batidas do coração, da roupa, e da voz. Uma pesquisa ${ }^{36}$ identificou que $99 \%$ das mães percebem as manifestações corporais dos bebês mediante o choro.

A educação e a habilidade de trabalhadores de saúde e dos pacientes, como ação que contribui com a ambiência hospitalar, foram aspectos considerados relevantes para os profissionais de enfermagem e pouco significantes para as usuárias. Em estudo $^{8}$, a reeducação da equipe de profissionais foi citada pelos pais como medida necessária para reduzir o ruído na UTIN. A ambiência oportuniza alternância nas relações de poder entre técnicos e usuários, especialmente quando os técnicos estão dispostos a participar do universo das experiências dos usuários ${ }^{37}$. 
No eixo relacionado à confortabilidade, o cuidado na ação de fechar portas e janelas foi considerado relevante para os profissionais e usuárias. O Ministério da Saúde recomenda o uso de abafadores em portas, pias, gavetas, lixeiras e superfícies para a manipulação dos materiais.

Profissionais de enfermagem revelaram apreço pela musicoterapia. Mães que têm filhos internados em um hospital cantam suas músicas preferidas aos pequenos, o que faz sentirem-se úteis aos filhos, mesmo quando eles estão em cuidados médicos intensivos $^{38}$. Os profissionais indicam também os alarmes luminosos, como estratégias da ambiência.

No eixo referente à gestão, profissionais consideram a manutenção dos materiais e equipamentos e a medição do ruído como ações que podem contribuir para a melhoria da ambiência. Já as usuárias consideram a manutenção dos equipamentos e das portas das enfermarias do AC. Estudo ${ }^{39}$ apresentou resultados antes e depois de reconstrução estrutural dentro de uma UTIN. Entre as medidas estruturais, foram realizadas as seguintes: redução de camas e aumento do espaçamento entre elas, localização de secretaria, posto de enfermagem e locais de solicitação de informações fora do berçário principal, instalação de ventilação e sistema de ar condicionado. Entre as medidas operacionais, utilizaram intervenções, como a educação pessoal, redução do volume de alarmes, utilização de plástico em vez de gavetas de metal e cestos de lixo, limitação de conversas perto do leito. Depois da reconstrução, os sons ainda se mantiveram elevados: indicam a necessidade de constante monitoramento.

Cabe refletir, finalmente, que o Alojamento Conjunto tem no verbo alojar, do francês loger, o significado de acolher. Os profissionais, por se considerarem corresponsáveis pelo bom andamento dos trabalhos, incluem a preocupação com o silêncio no âmbito do acolher, como primazia das ações relacionadas ao processo de trabalho a contribuir com a ambiência (eixo 1). O silêncio, intermeado com a musicoterapia e com os cuidados relacionados ao abrir/fechar de portas e janelas, entre as ações relativas à confortabilidade (eixo 2), providenciam os recursos humanizadores necessários, relacionados ao fato comum a todos: o nascimento, momento de fragilidade do qual depende o restante da vida.

Ao ingressarem temporariamente nessa ambiência, as usuárias deixam de lado aquela do seu cotidiano e passam a viver a pouca familiaridade com o local em que estão sendo cuidadas, onde os profissionais são desconhecidos, e vivem um turbilhão de sentimentos e emoções ${ }^{23}$. Isto as torna mais sensíveis aos ruídos, por misturarem dor e sofrimentos. E elas precisam do silêncio, pois estão vivendo um momento especial de suas vidas. Os ruídos, a exemplo das batidas das portas, são os que primeiro Ihe chamam a atenção e lhes provocam irritabilidade e dificuldade de concentração. Assim, indicam como ações relacionadas à gestão (terceiro eixo) a manutenção de materiais e das portas como ações para contribuir com a ambiência.

Os três eixos da ambiência, conforme o Ministério da Saúde ajudam na construção do espaço como ambiente terapêutico, por possibilitarem a reflexão e a maior participação dos sujeitos e por garantirem o bem-estar aos humanos em interação, com cheiros, cores, luzes e sons de paisagens sonoras; e por favorecerem a otimização dos recursos.

Finalizando com Heidegger, acolher é sinônimo de habitar, cujo significado é "resguardar". Parafraseando este filósofo em outras considerações sobre o habitar, se poderia dizer que, na ambiência do AC, os profissionais da enfermagem habitam (acolhem) à medida que salvam. Salvar não é somente livrar usuárias e recém-nascidos dos perigos da morte, doenças, complicações, etc., mas "deixar algo livre em seu próprio vigor" 40 , o que acontece quando gestantes, puérperas e recém-nascidos conseguem vivenciar a ambiência do bem-estar interior e da satisfação plena enquanto usuárias do sistema de saúde. Conduzir alguém a sua essência envolve dois verbos que têm sentido especial para os profissionais da enfermagem: aguardar a manifestação dos recém-nascidos em seus mistérios; e conduzi-los, com suas mães, ao desabrochar de si, compreendendo-se, ao mesmo tempo, como seres finitos e em constante processo de construção humana ${ }^{40}$.

Diante dos resultados deste estudo e diante da obrigação que os pesquisadores têm de apresentar os resultados à comunidade pesquisada, tendo em vista a obtenção de benefícios para ela, parece oportuno apresentar uma iniciativa que se some às demais propostas do Humaniza SUS, uma vez que os valores dos níveis de pressão sonora encontrados, neste trabalho estão acima do que é recomendado pelas instituições competentes. Recomenda-se:

a) Reunir as pesquisas já realizadas por enfermeiras da UFPR sobre o ruído hospitalar e programar apresentações aos gestores e profissionais;

b) Elaborar um programa de redução de ruído no $\mathrm{HC}$ que envolva a ambiência de cada setor, se possível contando com a assessoria de Fonoaudiólogos e Engenheira do Trabalho; 
c) Recomendar a prática da ambiência para os alunos no início de sua prática clínica nos hospitais.

d) Considerando a importância do tripé do projeto do Humaniza - SUS (gestor, profissionais e usuários) o processo de trabalho tem implicações na ambiência e na terapêutica dos usuários e demonstrou a importância de escutá-los. Além disso, demonstrou a pertinência das estratégias propostas: o ruído interfere na ambiência do serviço, gerando a necessidade de ações para correção das falhas apontadas.

\section{CONCLUSÃO}

Constatou-se nesse estudo que os níveis de ruído estão elevados em todas as áreas do Alojamento Conjunto. No entanto, tais níveis são insuficientes para causar perda auditiva. Também foi constatado que nem sempre as pessoas se percebem fazendo ruído.

Os profissionais de enfermagem percebem mais o ruído proveniente do ambiente externo, enquanto que as usuárias percebem mais o ruído interno. O ruído interfere na ambiência do Alojamento Conjunto, afetando negativamente as atividades diárias dos profissionais de enfermagem e a recuperação dos pacientes.

\section{ABSTRACT}

Purpose: to measure noise levels and investigate the perception of nursing professionals and users' health at a reference high-risk pregnancy maternity hospital in the city of Curitiba-PR. Methods: crosscut, quantitative study, held at a public hospital rooming-in, where 19 professionals and 77 users' perception was collected by means of interviews, while noise was assessed through an Integrating Bruel and Kjaer 2230 Meter, compensation A calibrated. Results: noise levels in all areas of the rooming-in are above the recommended in literature. (35 a $45 \mathrm{~dB}(\mathrm{~A})$ to the hospital). The higher levels were $67,7 \mathrm{~dB}(\mathrm{~A})$ at the nursing station and $65,3 \mathrm{~dB}(\mathrm{~A})$ at the visitors' room. Users and nursing professionals are exposed to nose sources from health team's work process, users and health team's behavior, and maternity external noise. Strategies to minimize noise levels and enhance rooming-in ambience are related to actions of the health team's work process, comfort and management. Conclusion: noise levels in the rooming-in are above the recommended. Nursing professionals perceive more external noise, while users perceive more internal noise. It was evidenced that high noise levels interfere with the rooming-in ambience and affect daily activities.

KEYWORDS: Obstetrics; Noise Effects; Rooming-in Care; Occupational Health

\section{REFERÊNCIAS}

1. Nogueira MFH, Piero KCD, Ramos EG, Souza MN, Dutra MVP. Mensuração de ruído sonoro em unidades neonatais e incubadoras com recém-nascidos: revisão sistemática de literatura. Rev. Latino-Am. Enfermagem [periódico na Internet]. 2011 [acesso em 25 jun 2011];19(1):10. Disponível em: <http://www.scielo.br/ scielo.php?script=sci_arttext\&pid=S0104$11692011000100028 \&$ Ing=en. $\quad$ http://dx.doi. org/10.1590/S0104-11692011000100028>

2. Peixoto PV, Araújo MAN, Kakehashi TY, Pinheiro EM. Nível de pressão sonora em Unidade de Terapia Intensiva Neonatal. Rev Esc Enferm USP [periódico na Internet]. 2011; [acesso em 20 Jan 2012];45(6):1309-14. Disponível em: http://www. scielo.br/scielo.php?script=sci_arttext\&pid=S0080- 62342011000600005\&lng=en. http://dx.doi. org/10.1590/S0080-62342011000600005.

3. Ichisato SMT, Scochi CGS. Ruídos na unidade de cuidado intensivo neonatal durante as passagens de plantão (enfermagem e/ou médica) e visita médica. Ciênc Cuidado Saúde. 2006;5(Suppl):127-33.

4. Zamberlan NE, Ichisato SMT, Rodarte MDO, Fujinaga $\mathrm{Cl}$, Hass VJ, Scochi CGS. Ruído em uma unidade de cuidado intermediário neonatal de um hospital universitário. Ciênc Cuid Saúde. 2008;7(4):431-8.

5. KomniskiTM, WatzlawickLF. Problemascausados pelo ruído no ambiente de trabalho. Revista Eletrônica Lato Sensu. 2007;2(1):147-60.

6. Souza KV, Tesin RR, Alves VH. Mães de recém-nascidos hospitalizados: em/entre círculos no processo de amamentação. Acta Paul Enferm. 2010;23(5):608-13. 
7. Perlman JM. Neurobehavioral deficits in premature graduates of intensive care: potential medical and neonatal environmental risk factors. Pediatrics. 2001;108:1339-48.

8. Aurélio FS, Tochetto TM. Ruído em uma unidade de terapia intensiva neonatal: mensuração e percepção de profissionais e pais. Revista Paulista de Pediatria. 2010;28(2):162-9.

9. Menon D, Martins AP, Dyniewicz AM. Condições de conforto do paciente internado em UTI neonatal. Cad Esc Saude Enferm. 2008;(1):1-15.

10. Tamez RN, Silva MJP. Enfermagem na uti neonatal: assistência ao recém-nascido de risco. $3^{\text {a }}$ ed. Rio de Janeiro: Guanabara Koogan; 2002.

11. Uchôa NT, Procianoy RS, Lavinsky L, Sleifer P. Prevalência de perda auditiva em recém-nascidos de muito baixo peso. J Pediatria. 2003;79(2):123-8.

12. Oliveira EB, Lisboa MTL. Exposição ao ruído tecnológico em CTI: estratégias coletivas de defesa dos trabalhadores de enfermagem. Esc Anna Nery. Rev Enferm. 2009;13(1):24-30.

13. Carvalho WB, Pedreira MLG, Aguiar MAL. Nível de ruídos em uma unidade de cuidados intensivos pediátricos. J. Pediatr. [periódico na Internet] 2005 [acesso em 25 Jan 2011];81(6):495-8.

Disponível em: http://www.scielo.br/ scielo.php?script=sci_arttext\&pid=S002175572005000800015\&lng=en. http://dx.doi. org/10.1590/S0021-75572005000800015.

14. Assossiação Brasileira de Enfermagem. Cartilha do Trabalhador de Enfermagem - Saúde, segurança e boas condições de trabalho / ABEn/RJ - Rio de Janeiro, 2006. Disponível em <http://bvsms.saude. gov.br/bvs/publicacoes/cartilha_aben.pdf>. Acesso em 09 fev 2011.

15. Hartoon JC, Treuting EG. Is noise a potential hazard to pregnancy? Occup Health Nurs. 1981;29(1):20-3.

16. Quick CT, Lapertosa BJ. Contribuição ao estudo das alterações auditivas e de ordem neurovegetativas atribuíveis ao ruído. Revista Brasileira de Saúde Ocupacional. 1981;(36):50-6.

17. Macedo ISC, Mateus DC, Costa EMGC, Aspirino ACL, Lourenço EA. Avaliação do ruído em unidades de terapia intensiva. Brazilian Journal of Otorhinolaryngology. 2009;75(6):844-6.

18. Nurminen T. Female noise exposure, shift work and reproduction. J. Occup. Environ. Med. [S. I.] 1995;37(8):945-50.

19. O que é Humaniza SUS. Disponível em:< http://portal.saude.gov.br/portal/saude/cidadao/ visualizar_texto.cfm?idtxt=28288>. Acesso em: 2 maio 2011.

20. Brasil. Ambiência. Brasília - DF, 2004.
21. NR 15. Atividades e operações insalubres. Diário Oficial da união. Portaria MTb n. 3.214 , de 8 de junho de 1978.

22. Diniz D, Medeiros M. Aborto no Brasil: uma pesquisa domiciliar com técnica de urna. Ciênc Saúde Rev Saúde Pública. 2011;45(5):981-5.

Disponível em <http://www.scielo.br/ scielo.php? script $=$ sci_arttext\&pid $=$ S1413$81232010000700002 \& \operatorname{lng}=e n \& n r m=i s o>$. Acesso em 01 Dez. 2011.

23. Frello AT, Carraro TE. Conforto no Processo de Parto sob a Perspectiva das Puérperas. Revista Enfermagem UERJ. 2010;18(3):441-5.

24. TiensoliLO, GoulartLMHF, ResendeLM,Colosimo EA. Triagem auditiva em hospital público de Belo Horizonte, Minas Gerais, Brasil: deficiência auditiva e seus fatores de risco em neonatos e lactentes. Cad. Saúde Pública [periódico na Internet].2007 [acesso em 13 de agosto de 2010];23(6):1431-41. Disponível em: http://www.scielosp.org/ scielo.php?script $=$ sci_arttext $\&$ pid $=$ S0102$311 \times 2007000600018 \&$ Ing $=$ en. http://dx.doi. org/10.1590/S0102-311X2007000600018.

25. Barros AJD, Santos IS, Matijasevich A, Domingues MR, Silveira M, Barros FC et al. Padrões dos partos em uma corte de nascimentos: cesarianas quase universais para os ricos. Rev. Saúde Pública [periódico na internet]. 2011. [acesso em 30 nov. 2011];45(4):635-43.

Disponível em:< http://dx.doi.org/10.1590/ S0034-89102011005000039>.

26. Vega CEP, Kahhale S, Zugaib M. Maternal mortality due to arterial hypertension in São Paulo City (1995-1999). Clinics. [periódico na Internet]. 2007 [acesso em o1 de dez 2010];62(6):679-84.

Disponível em: http://www.scielo.br/ scielo.php?script $=$ sci_arttext\&pid=S180759322007000600004\&lng=en. http://dx.doi. org/10.1590/S1807-59322007000600004.

27. Oliveira MIV, Bezerra MGA, Filho JGB, Veras MAC, Bezerra, JP. Perfil de mães e recém-nascidos na presença do diabetes mellitus. Rev. Rene. 2009;10(4):28-36.

Disponível em:< http://132.248.9.1:8991/hevila/ RevistaRENE/2009/vol10/no4/2.pdf>. Acesso em 30 nov. 2011.

28. Soares CP, Marques LR, Flores NGC. Triagem auditiva neonatal: aplicabilidade clínica na rotina dos médicos pediatras neonatologistas. Rev. CEFAC. 2008;10(1): 110-6.

Disponível em <http://www.scielo.br/ scielo.php? script $=$ sci_arttext \&pid $=$ S1516$18462008000100015 \&$ Ing $=e n \& n r m=i s o>$. Acesso em 03 Dez. 2011. 
29. Borges MMR, Cardoso MVLML, Chaves EMC, Bezerra MGA. Som e barulho no ambiente da unidade neonatal. Pediatra Atual. 2007;20(2):6-9. 30.Cardoso MVLML, Chaves EMC, Bezerra MGA. Ruídos e barulhos na unidade neonatal. Revista Brasileira de Enfermagem. 2010;63(4):561-6.

31. Gaíva MAM, Marquesi M, Rosa MKO. O sono do recém-nascido internado em unidade de terapia intensiva: cuidados de enfermagem. Ciência, Cuidado \& Saúde. 2010;9(3):602-9.

32. Corrêa AL. Ruído: níveis de pressão sonora captados no interior e exterior de incubadora em unidades de cuidados intensivos neonatal [dissertação]. São José dos Campos (SP): Universidade do Vale do Paraíba; 2005.

33. Beserra FM, Souza AM, Moreira DA, Alves MDS, D'Alencar BP. Significado do trabalho dos profissionais de enfermagem no hospital geral. Avances em Enfermería. 2010;28(2):31-9.

34. Lipp MN, Rocha JC. Stress, hipertensão e qualidade de vida. Campinas: Papirus, 1994.

35. Royas ADV, Marziale MHP. A situação de trabalho do pessoal de enfermagem no contexto de um hospital argentino: um estudo sob a ótica da ergonomia. Rev. latino-am. Enfermagem. 2011;9(1):102-8.
36. Zunino SM, Ficanha TB. A dor do recémnascido: percepção materna na unidade de alojamento conjunto. [Monografia] (Enfermagem): Universidade do Vale do Itajaí; Biguaçu, 2008.

37. Silva LV. Ambiência: conviver produz saúde? TCC - (Especialista em Saúde Mental) - Grupo Hospitalar Conceição. S. I., 2008. Disponível em:< http://coleciona-sus.bvs.br/lildbi/ docsonline/2/4/142-TRCAmbi\%EAncia.pdf>. Acesso em 30 nov. 2011.

38. Karst LT. Musicoterapia com mães de recémnascidos internados em UTI neonatal. 2004, 72 f. [Monografia]: Curso de Musicoterapia - Escola de Música e Artes Cênicas da Universidade Federal de Goiás. Goiânia, 2004. Disponível em:< http://www. sgmt.com.br/musicoterapiamaesutineonatal_lara_ karst.pdf>. Acesso em: 30 nov. 2011.

39. Krueger C, Schue S, Parker L. Neonatal intensive care unit. MCN. 2007;32(6) 359-62. Disponivel em:http://www.nursingcenter.com/pdf. asp?AID=750763>. Acesso em 30 nov. 2011.

40. Heidegger M. Construir, habitar, pensar. In: Ensaios e Conferências. Tradução: de Emmanuel Carneiro Leão, Gilvan Fogel, Márcia Sá Cavalcante Schuback. Petrópolis: Vozes, 2002, p. 130.

Recebido em: 17/04/2012

Aceito em: 29/11/2012

Endereço para correspondência:

Liane Fuhr Pivatto

Rua Vitório Sbalqueiro 670

Curitiba - PR

CEP: 82100-060

E-mail: liane_hc@ @otmail.com 